\title{
CORRECTION
}

\section{Correction to: Pain Trajectories Following Subarachnoid Hemorrhage are Associated with Continued Opioid Use at Outpatient Follow-Up}

Matthew N. Jaffa ${ }^{1,2}$, Ruchira M. Jha 3,4,5,6,7, Jonathan Elmer ${ }^{3,4,8}$, Adam Kardon', Jamie E. Podell 1,2, Benjamin E. Zusman ${ }^{4}$, Madeleine C. Smith', J. Marc Simard9,10,11 , Gunjan Y. Parikh',2, Michael J. Armahizer ${ }^{12}$, Neeraj Badjatia ${ }^{1,2}$ and Nicholas A. Morris ${ }^{1,2^{*}}$ (I)

(c) 2021 Springer Science+Business Media, LLC, part of Springer Nature and Neurocritical Care Society

\section{Correction to:}

\section{Neurocrit Care}

https://doi.org/10.1007/s12028-021-01282-5

This article was updated to correct Fig. 3.

\section{Author details}

${ }^{1}$ Department of Neurology, School of Medicine, University of Maryland, Baltimore, MD, USA. ${ }^{2}$ Program in Trauma, R Adams Cowley Shock Trauma Center, Baltimore, MD, USA. ${ }^{3}$ Department of Critical Care Medicine, School of Medicine, University of Pittsburgh, Pittsburgh, PA, USA. ${ }^{4}$ Department of Neurology, School of Medicine, University of Pittsburgh, Pittsburgh, PA, USA. ${ }^{5}$ Department of Neurosurgery, School of Medicine, University of Pittsburgh, Pittsburgh, PA, USA. ${ }^{6}$ Safar Center for Resuscitation Research, School of Medicine, University of Pittsburgh, Pittsburgh, PA, USA. ${ }^{7}$ Clinical and Translational Science Institute,
School of Medicine, University of Pittsburgh, Pittsburgh, PA, USA. ${ }^{8}$ Department of Emergency Medicine, School of Medicine, University of Pittsburgh, Pittsburgh, PA, USA. ${ }^{9}$ Department of Neurosurgery, School of Medicine, University of Maryland, Baltimore, MD, USA. ${ }^{10}$ Department of Pathology, School of Medicine, University of Maryland, Baltimore, MD, USA. ${ }^{11}$ Department of Physiology, School of Medicine, University of Maryland, Baltimore, MD, USA. ${ }^{12}$ Department of Pharmacy Services, University of Maryland Medical Center, Baltimore, MD, USA.

\section{Publisher's Note}

Springer Nature remains neutral with regard to jurisdictional claims in published maps and institutional affiliations.

Published online: 18 October 2021

*Correspondence: nicholas.morris@som.umaryland.edu

1 Department of Neurology, School of Medicine, University of Maryland, Baltimore, MD, USA

Full list of author information is available at the end of the article 